PROCEEDINGS OF THE

AMERICAN MATHEMATICAL SOCIETY

Volume 131, Number 4, Pages 1243-1250

S 0002-9939(02)06669-8

Article electronically published on September 5, 2002

\title{
FERENC LUKÁCS TYPE THEOREMS IN TERMS OF THE ABEL-POISSON MEAN OF CONJUGATE SERIES
}

\author{
FERENC MÓRICZ
}

(Communicated by Andreas Seeger)

\begin{abstract}
A theorem of Ferenc Lukács determines the generalized jumps of a periodic, Lebesgue integrable function $f$ in terms of the partial sum of the conjugate series to the Fourier series of $f$. The main aim of this paper is to prove an analogous theorem in terms of the Abel-Poisson mean. We also prove an estimate of the partial derivative (with respect to the angle) of the Abel-Poisson mean of an integrable function $F$ at those points at which $F$ is smooth. Finally, we reveal the intimate relation between these two results.
\end{abstract}

\section{A theorem of Ferenc LukÁcs}

Let $f$ be a periodic, Lebesgue integrable function, in symbol: $f \in L^{1}(\mathbb{T}), \mathbb{T}:=$ $[-\pi, \pi)$, with Fourier series

$$
f(x) \sim \frac{1}{2} a_{0}+\sum_{k=1}^{\infty}\left(a_{k} \cos k x+b_{k} \sin k x\right),
$$

where

$$
a_{k}:=\frac{1}{\pi} \int_{\mathbb{T}} f(x) \cos k x d x \quad \text { and } \quad b_{k}:=\frac{1}{\pi} \int_{\mathbb{T}} f(x) \sin k x d x
$$

are the Fourier coefficients of $f$. We recall that the conjugate series to (1.1) is defined by

$$
\sum_{k=1}^{\infty}\left(a_{k} \sin k x-b_{k} \cos k x\right) .
$$

Denote by $\tilde{s}_{n}(f, x)$ the $n$th partial sum of series (1.3). The following theorem was proved by Ferenc Lukács [2].

Received by the editors June 21, 2001 and, in revised form, December 3, 2001.

2000 Mathematics Subject Classification. Primary 42A50, 42A16.

Key words and phrases. Function of bounded variation, induced Borel measure, Fourier series, theorem of Fejér, conjugate series, generalized jump, theorem of Ferenc Lukács, Abel-Poisson mean, smoothness, Zygmund classes $\lambda_{*}$ and $\Lambda_{*}$.

This research was started during the author's visit to the Université de Paris-Sud, Orsay, in May 2001, and it was partially supported by the Hungarian National Foundation for Scientific Research under Grant T 029094. 
Theorem 1. Let $f \in L^{1}(\mathbb{T})$ and $x \in \mathbb{T}$. If there exists a number $d_{x}(f)$ such that

$$
\lim _{h \rightarrow 0+} \frac{1}{h} \int_{0}^{h}\left|[f(x+t)-f(x-t)]-d_{x}(f)\right| d t=0,
$$

then

$$
\lim _{n \rightarrow \infty} \frac{\tilde{s}_{n}(f, x)}{\log n}=-\frac{1}{\pi} d_{x}(f)
$$

By 'log' we mean the natural logarithm.

It is clear that if the finite limit

$$
d_{x}(f):=\lim _{t \rightarrow 0+}[f(x+t)-f(x-t)]
$$

exists, then condition (1.4) is also satisfied with the same $d_{x}(f)$. In particular, if a periodic function $f$ is of bounded variation over $[-\pi, \pi]$, then (1.5) is satisfied at every point $x \in \mathbb{T}$ with

$$
d_{x}(f):=f(x+0)-f(x-0) .
$$

This means that the terms of the Fourier series of $f$ determine the (ordinary) jumps of $f$ at any point $x \in \mathbb{T}$ of discontinuity of first kind. Or equivalently, the terms of the Fourier series of $f$ determine the atoms of the finite Borel measure induced by $f$ on $\mathbb{T}$.

We note that Fejér [1] achieved the first result in the subject of determining the jumps of a function of bounded variation in terms of the partial sum of its Fourier series. Then Zygmund [4 p. 108] proved a Fejér type theorem via the Abel-Poisson mean of the Fourier series in question.

\section{MAIN RESUlts}

One of the aims of the present paper is to prove a Ferenc Lukács type theorem in terms of the Abel-Poisson mean of the conjugate series (1.3). We recall that the Abel-Poisson mean of (1.3) is defined by

$$
\tilde{f}(r, x):=\sum_{k=1}^{\infty}\left(a_{k} \sin k x-b_{k} \cos k x\right) r^{k}, \quad 0 \leq r<1 .
$$

The analogue of Theorem 1 reads as follows.

Theorem 2. If $f \in L^{1}(\mathbb{T})$ and the finite limit

$$
\delta_{x}(f):=\lim _{h \rightarrow 0+} \frac{1}{h} \int_{0}^{h}[f(x+t)-f(x-t)] d t
$$

exists at some point $x \in \mathbb{T}$, then

$$
\lim _{r \rightarrow 1-} \frac{\tilde{f}(r, x)}{\log (1-r)}=\frac{1}{\pi} \delta_{x}(f) .
$$

The quantity $\delta_{x}(f)$ defined in (2.2) may be called the generalized jump of the function $f$ at the point $x$. The existence of $d_{x}(f)$ in (1.6) or even in (1.4) implies that of $\delta_{x}(f)$, and both numbers are equal. Observe the lack of the absolute value bars in (2.2). Loosely speaking, condition (2.2) expresses a differentiability property of an integral, while (1.4) resembles the definition of a Lebesgue point. 
An immediate corollary of Theorem 2 is that if a periodic function $f$ is of bounded variation over $[-\pi, \pi]$, then at every point $x \in \mathbb{T}$ we have

$$
\lim _{r \rightarrow 1-} \frac{\tilde{f}(r, x)}{\log (1-r)}=\frac{1}{\pi}[f(x+0)-f(x-0)] .
$$

We remind the reader that a function $F$ is said to be smooth at some inner point $x$ of the domain of $F$ if the following limit relation holds:

$$
\Delta(F, x, h):=\frac{1}{h}[F(x+h)+F(x-h)-2 F(x)] \rightarrow 0 \quad \text { as } \quad h \rightarrow 0 .
$$

The function class $\lambda_{*}(\mathbb{T})$ is defined to consist of all periodic, continuous functions $F$ such that (2.4) holds uniformly in $x \in \mathbb{T}$. The class $\Lambda_{*}(\mathbb{T})$ is defined by the requirement that the ratio $\Delta(F, x, h)$ is uniformly bounded in $x \in \mathbb{T}$ and $h>0$. These function classes (not only in the periodic case) were introduced by Zygmund [3] (see also [4, p. 43]).

Our second main result is the following

Theorem 3. (i) If a function $F \in L^{1}(\mathbb{T})$ is smooth at some point $x \in \mathbb{T}$, then for the Abel-Poisson mean $\tilde{F}(r, x)$ of the conjugate series to the Fourier series of $F$ we have

$$
\frac{\partial \tilde{F}(r, x)}{\partial x}=o\left\{\log \frac{1}{1-r}\right\} \quad \text { as } \quad r \rightarrow 1-.
$$

(ii) If $F \in \lambda_{*}(\mathbb{T})$, then (2.5) holds uniformly in $x \in \mathbb{T}$. If $F \in \Lambda_{*}(\mathbb{T})$, then (2.5) holds with ' $O$ ' in place of ' $O$ ', uniformly in $0 \leq r<1$ and $x \in \mathbb{T}$.

We note that Theorem 3 can be considered as a counterpart of a theorem of Zygmund [4, p. 109] which provides an estimate of $\partial^{2} F(r, x) / \partial x^{2}$, where by $F(r, x)$ we denote the Abel-Poisson mean of the Fourier series of $F$.

\section{Auxiliary results on the Conjugate Abel-Poisson kernel}

We start with the representation

$$
\tilde{f}(r, x)=\frac{1}{\pi} \int_{\mathbb{T}} f(x-t) Q(r, t) d t
$$

where

$$
Q(r, t):=\sum_{k=1}^{\infty} r^{k} \sin k t=\frac{r \sin t}{1-2 r \cos t+r^{2}}, \quad 0 \leq r<1,
$$

is the conjugate Abel-Poisson kernel. (See, for example, [4, p. 96].)

Since $Q(r, t)$ is odd in $t$, from (3.1) it follows that

$$
\tilde{f}(r, x)=\frac{1}{\pi} \int_{0}^{\pi}[f(x-t)-f(x+t)] Q(r, t) d t, \quad 0 \leq r<1 .
$$

The other crucial property is that $Q(r, t)$ is positive for $0<t<\pi$. From (3.2) it also follows that

$$
Q(r, t)=\frac{r \sin t}{(1-r)^{2}+4 r \sin ^{2} \frac{t}{2}} \leq \frac{1}{2 \tan \frac{t}{2}}, \quad 0 \leq r<1 \quad \text { and } \quad 0<t<\pi .
$$

Lemma 1. For $0 \leq r<1$, we have

$$
Q_{r}:=\int_{0}^{\pi} Q(r, t) d t=\log \frac{1+r}{1-r} .
$$


Proof. By elementary calculus, we have

$$
Q_{r}=\left[\frac{1}{2} \log \left\{(1-r)^{2}+4 r \sin ^{2} \frac{t}{2}\right\}\right]_{t=0}^{\pi},
$$

which gives the right-hand side in (3.5).

Next, we examine the partial derivative

$$
Q^{\prime}(r, t):=\frac{\partial Q(r, t)}{\partial t}=\frac{r\left(1+r^{2}\right) \cos t-2 r^{2}}{\left(1-2 r \cos t+r^{2}\right)^{2}} .
$$

It is clear that $Q^{\prime}(r, t)$ changes sign in the interval $(0, \pi)$ only once, namely, for $t=\tau=\tau(r)$ satisfying

$$
\cos \tau=\frac{2 r}{1+r^{2}},
$$

so that $\tau \rightarrow 0+$ as $r \rightarrow 1-$. Since $0<\tau \leq \pi / 2$ for $0 \leq r<1$, we have

$$
1-\frac{\tau^{2}}{2} \leq \frac{2 r}{1+r^{2}},
$$

whence

$$
\tau \geq \sqrt{2} \frac{1-r}{\sqrt{1+r^{2}}} \geq 1-r .
$$

Lemma 2. For $0 \leq r<1$, we have

$$
\int_{0}^{\pi} t\left|Q^{\prime}(r, t)\right| d t \leq 2+\log \frac{\pi}{1-r} .
$$

Proof. By integration by parts, we obtain

$$
\begin{gathered}
\int_{0}^{\pi} t\left|Q^{\prime}(r, t)\right| d t=\int_{0}^{\tau} t Q^{\prime}(r, t) d t-\int_{\tau}^{\pi} t Q^{\prime}(r, t) d t \\
=2 \tau Q(r, \tau)-\int_{0}^{\tau} Q(r, t) d t+\int_{\tau}^{\pi} Q(r, t) d t .
\end{gathered}
$$

By virtue of (3.4) and (3.7), we conclude that

$$
\begin{aligned}
\int_{0}^{\pi} t\left|Q^{\prime}(r, t)\right| d t & \leq \frac{2 \tau \sin \tau}{4 \sin ^{2} \frac{\tau}{2}}+\int_{\tau}^{\pi} \frac{\sin t}{4 \sin ^{2} \frac{t}{2}} d t \\
& =\frac{\tau}{\tan \frac{\tau}{2}}+\int_{\tau}^{\pi} \frac{d t}{2 \tan \frac{t}{2}},
\end{aligned}
$$

whence (3.8) follows immediately.

\section{Proof of Theorem 2}

By Lemma 1, we have

$$
\lim _{r \rightarrow 1-} \frac{Q_{r}}{-\log (1-r)}=1 .
$$

Thus, it is enough to prove that

$$
\lim _{r \rightarrow 1-}\left(\frac{\tilde{f}(r, x)}{Q_{r}}+\frac{1}{\pi} \delta_{x}(f)\right)=0 .
$$


By (2.2), given any $\varepsilon>0$ we can choose $0<\eta<\pi / 2$ so that for the function

$$
I(h):=\int_{0}^{h}\left[f(x-t)-f(x+t)+\delta_{x}(f)\right] d t
$$

we have

$$
|I(h)| \leq \varepsilon h \quad \text { if } \quad 0 \leq h \leq \eta
$$

Keeping (3.3), the notation in (3.5), and (4.2) in mind, we may write that

$$
\begin{gathered}
\frac{\tilde{f}(r, x)}{Q_{r}}+\frac{1}{\pi} \delta_{x}(f)=\frac{1}{\pi Q_{r}} \int_{0}^{\pi}\left[f(x-t)-f(x+t)+\delta_{x}(f)\right] Q(r, t) d t \\
=\frac{1}{\pi Q_{r}}\left(\int_{0}^{\eta}+\int_{\eta}^{\pi}\right)=: J_{1}+J_{2}, \quad \text { say. }
\end{gathered}
$$

First, integrating by parts and making use of (4.2), (3.4), (3.5) and (3.8) yield

$$
\begin{aligned}
\left|J_{1}\right| & \leq \frac{1}{\pi Q_{r}}\left(I(\eta) Q(r, \eta)+\int_{0}^{\eta}\left|I(t) Q^{\prime}(r, t)\right| d t\right) \\
& \leq \frac{\varepsilon}{\pi Q_{r}}\left(\eta Q(r, \eta)+\int_{0}^{\eta} t\left|Q^{\prime}(r, t)\right| d t\right) \\
& \leq \frac{\varepsilon}{-\pi \log (1-r)}\left\{\frac{\eta}{2 \tan \frac{\eta}{2}}+2+\log \pi-\log (1-r)\right\} \\
& \leq \frac{\varepsilon}{-\pi \log (1-r)}\left\{\frac{9}{2}-\log (1-r)\right\} \leq \frac{11 \varepsilon}{2 \pi} \quad \text { if } \quad r \geq \frac{e-1}{e},
\end{aligned}
$$

since $\log \pi \leq 3 / 2$ and $-\log (1-r) \geq 1$ if $0<(1-r) \leq 1 / e$.

Second, by (3.4) and (3.5) we obtain

$$
\begin{aligned}
\left|J_{2}\right| & \leq \frac{1}{\pi Q_{r}} \int_{\eta}^{\pi}\left|f(x-t)-f(x+t)+\delta_{x}(f)\right| Q(r, t) d t \\
& \leq \frac{1}{\pi Q_{r}} \frac{1}{2 \tan \frac{\eta}{2}}\left(\int_{\mathbb{T}}|f(t)| d t+2 \pi\left|\delta_{x}(f)\right|\right) \\
& =O\left\{\frac{1}{-\log (1-r)}\right\} \quad \text { as } \quad r \rightarrow 1-.
\end{aligned}
$$

Combining (4.3)-(4.5) gives (4.1), which is equivalent to (2.3) which was to be proved.

\section{Proof of Theorem 3}

Part (i). We make use of (3.1) with $F$ in place of $f$ and the series representation of $Q(r, t)$ in (3.2) (which allows a term-by-term differentiation) to obtain

$$
\frac{\partial \tilde{F}(r, x)}{\partial x}=\frac{1}{\pi} \int_{\mathbb{T}} F(t) Q^{\prime}(r, x-t) d t=\frac{1}{\pi} \int_{\mathbb{T}} F(x-t) Q^{\prime}(r, t) d t .
$$

Since $Q^{\prime}(r, t)$ is even in $t$ (see (3.6)) and

$$
\int_{0}^{\pi} Q^{\prime}(r, t) d t=Q(r, \pi)-Q(r, 0)=0,
$$

from (5.1) we conclude that

$$
\frac{\partial \tilde{F}(r, x)}{\partial x}=\frac{1}{\pi} \int_{0}^{\pi}[F(x+t)+F(x-t)-2 F(x)] Q^{\prime}(r, t) d t, \quad 0 \leq r<1 .
$$


By assumption, $F$ is smooth at $x$. Thus, given any $\varepsilon>0$ there exists $\eta>0$ so that (cf. (2.4) as to the notation)

$$
|t \Delta(F, x, t)|=|F(x+t)+F(x-t)-2 F(x)| \leq \varepsilon t \quad \text { if } \quad 0 \leq t \leq \eta .
$$

Accordingly, we decompose the integral in (5.2) as follows:

$$
\begin{gathered}
\frac{\partial \tilde{F}(r, x) / \partial x}{-\log (1-r)}=\frac{1}{-\pi \log (1-r)}\left(\int_{0}^{\eta}+\int_{\eta}^{\pi}\right) t \Delta(F, x, t) Q^{\prime}(r, t) d t \\
=: I_{1}+I_{2}, \quad \text { say. }
\end{gathered}
$$

By (5.3) and (3.8), we have

$$
\left|I_{1}\right| \leq \frac{1}{-\pi \log (1-r)} \int_{0}^{\eta} \varepsilon\left|t Q^{\prime}(r, t)\right| d t=O(\varepsilon) \quad \text { as } \quad r \rightarrow 1-.
$$

On the other hand, we estimate $\left|Q^{\prime}(r, t)\right|$ (see (3.6)) in the same way as in the case of inequality (3.4) to obtain

$$
\left|Q^{\prime}(r, t)\right| \leq \frac{1}{4 r \sin ^{4} \frac{t}{2}}, \quad 0 \leq r<1 \quad \text { and } \quad 0<t \leq \pi .
$$

Taking into account this and the first equality in (5.3) gives

$$
\begin{aligned}
\left|I_{2}\right| & \leq \frac{1}{-\pi \log (1-r)} \frac{1}{4 r \sin ^{4} \frac{\eta}{2}} \int_{\eta}^{\pi}|F(x+t)+F(x-t)-2 F(x)| d t \\
& \leq \frac{1}{-\pi \log (1-r)} \frac{1}{4 r \sin ^{4} \frac{\eta}{2}}\left(\int_{\mathbb{T}}|F(t)| d t+2 \pi|F(x)|\right) \\
& =O\left\{\frac{1}{-\log (1-r)}\right\} \quad \text { as } \quad r \rightarrow 1-.
\end{aligned}
$$

Combining (5.4)-(5.6) yields (2.5) to be proved.

Part (ii). The obvious extension to uniformity completes the proof of the first statement when $F \in \lambda_{*}(\mathbb{T})$. The proof of the second statement when $F \in \Lambda_{*}(\mathbb{T})$ runs along the same lines, the details of which are left to the reader.

\section{Concluding Remarks}

Remark 1. We start with a function $f \in L^{1}(\mathbb{T})$. Being interested in the AbelPoisson mean of the conjugate series to the Fourier series of $f$, there is no loss of generality if we assume that $a_{0}=0$ in (1.2). Then the integral $F$ of $f$ defined by

$$
F(u):=\int_{0}^{u} f(t) d t, \quad u \in \mathbb{R},
$$

is also periodic. Furthermore, we consider such a point $x \in \mathbb{T}$ at which $\delta_{x}(f)=0$.

We claim that if we apply Theorem 3 to this $F$ at this point $x$, then conclusion (2.5) coincides with (2.3). In fact, by (6.1), condition (2.2) can be rewritten in the form

$$
\delta_{x}(f)=\lim _{h \rightarrow 0+} \frac{1}{h}[F(x+h)+F(x-h)-2 F(x)],
$$

whence we see that $\delta_{x}(f)=0$ is the same thing as the smoothness of $F$ at $x$. 
On the other hand, if $A_{k}$ and $B_{k}$ are the Fourier coefficients of $F$, then an integration by parts gives (see, for example, [4 p. 42]) that

$$
A_{k}=-\frac{b_{k}}{k} \quad \text { and } \quad B_{k}=\frac{a_{k}}{k}, \quad k=1,2, \ldots
$$

Hence it follows immediately that

$$
\frac{\partial \tilde{F}(r, x)}{\partial x}=\tilde{f}(r, x), \quad 0 \leq r<1 .
$$

This justifies our claim made above.

In other words, we have found another proof of Theorem 2 by means of Theorem 3 in the particular case when $\delta_{x}(f)=0$.

Remark 2. To prove Theorem 2 in the general case when $\delta_{x}(f) \neq 0$, we could have proceeded in the following way, as well. Imitating the argument of Fejér in [1, we introduce a new function $g$ by setting

$$
g(\xi):=f(\xi)-\frac{1}{\pi} \delta_{x}(f) \phi(\xi-x), \quad \xi \in \mathbb{T},
$$

where the auxiliary function $\phi$ is defined by

$$
\phi(t):=\frac{1}{2}(\pi-t) \quad \text { for } \quad 0<t<2 \pi,
$$

$\phi(0)=\phi(2 \pi):=0$, and $\phi$ is continued periodically for all $t \in \mathbb{R}$. Since the ordinary jump of the function $\phi(\xi-x)$ at the point $\xi:=x$ equals $\pi$, we have $\delta_{x}(g)=0$. By Remark 1, we conclude that

$$
\lim _{r \rightarrow 1-} \frac{\tilde{s}_{n}(g, x)}{\log (1-r)}=0
$$

By (6.2), it remains only to take into account that the Fourier series of $\phi(\xi-x)$ (as a function of $\xi$ ) and its conjugate series are given by

$$
\phi(\xi-x) \sim \sum_{k=1}^{\infty} \frac{\sin k(\xi-x)}{k} \quad \text { and }-\sum_{k=1}^{\infty} \frac{\cos k(\xi-x)}{k},
$$

respectively. Hence it follows immediately that

$$
\frac{\tilde{\phi}(\cdot-x)(r, x)}{\log (1-r)}=-\frac{1}{\log (1-r)} \sum_{k=1}^{\infty} \frac{r^{k}}{k}=1, \quad 0<r<1 .
$$

Combining (6.2)-(6.4) provides (2.3) in the general case when $\delta_{x}(f) \neq 0$.

Remark 3. In case $x:=0,(6.4)$ is of the form

$$
\frac{\tilde{\phi}(r, 0)}{\log (1-r)}=1=\frac{1}{\pi} d_{0}(\phi), \quad 0<r<1,
$$

where $d_{0}(\phi)$ is defined in (1.6). This shows that relation (2.3) holds true even without " $\lim _{r \rightarrow 1-}$ " in the case of the function $\phi(t)$ defined above (observe that this time $\left.\delta_{0}(\phi)=d_{0}(\phi)\right)$. 


\section{REFERENCES}

1. L. Fejér, Über die Bestimmung des Sprunges der Funktion aus ihrer Fourierreihe, J. reine angew. Math. 142 (1913), 165-188.

2. F. Lukács, Über die Bestimmung des Sprunges einer Funktion aus ihrer Fourierreihe, J. reine angew. Math. 150 (1920), 107-112.

3. A. Zygmund, Smooth functions, Duke J. 12 (1945), 47-76. MR 7:60b

4. A. Zygmund, Trigonometric series, Vol. 1, Cambridge Univ. Press, Cambridge, UK, 1959. MR 21:6498

Bolyai Institute, University of Szeged, Aradi Vértanúk Tere 1, 6720 Szeged, Hungary

E-mail address: moricz@math.u-szeged.hu 